$\underline{\text { Review Article }}$

\title{
CURRENT STATUS OF MATERIOVIGILANCE GLOBALLY-AN UTTER OVERVIEW WITH CLINICAL CASE PERUSAL
}

\author{
PRATIBHA CHAUHAN ${ }^{1}$, AALIA ZARREEN ${ }^{1}$, MOHAMMAD KASHIF IQUBAL ${ }^{2 *}$
}

${ }^{1}$ Department of Pharmacology, School of Pharmaceutical Education and Research, Jamia Hamdard, New Delhi 110062, India. ${ }^{2}$ Department of Pharmaceutics, School of Pharmaceutical Education and Research, Jamia Hamdard, New Delhi 110062, India

Email: mkashifiqubal@ymail.com

Received: 27 Jun 2019, Revised and Accepted: 02 Sep 2019

\section{ABSTRACT}

Today's medical devices are the lifesaving tool and these tools are very frequently used globally since long ago. With the increase in the number of medical devices and their uses, there is an increase in the number of adverse events related to such devices. That is why Materiovigilance is an important tool for the identification, collection, reporting, and analysis of any aversive catastrophe related to the use of medical devices and protection of a patient's life by fending its reiteration. Post marketing surveillance of medical devices has been initiated in many countries, like the United States of America has started a program under the name Medical Device Reporting (MDR), France, Australia and the United Kingdom have also come up with their own programs. Apart from this India has also initiated the Materiovigilance program by Drug Controller General of India (DCGI) at the Indian Pharmacopoeia Commission (IPC) in Ghaziabad in 2015. In this article we have discussed about the risks associated with the use of different classes of medical devices and the need of Materiovigilance program. This article also discusses the adverse events associated with the medical devices, the reporting criteria of those adverse events and the different clinical events of the medical devices.

Keywords: Medical device, Materiovigilance, Regulations, Clinical case studies

(C) 2019 The Authors. Published by Innovare Academic Sciences Pvt Ltd. This is an open access article under the CC BY license (http://creativecommons.org/licenses/by/4.0/] DOI: http://dx.doi.org/10.22159/ijpps.2019v11i10.34716

\section{INTRODUCTION}

The term "vigilance" means close monitoring of the possible adverse effects [1-4]. Materiovigilance is the study of adverse events associated with the use of medical devices. It deals with the close monitoring of medical devices after post-marketing phase $[1,5]$. The term "medical device" has been defined by the World Health Organization (WHO) as any instrument, apparatus, reagent for in vitro use, implant, device for tissue cutting or wound covering, highly sophisticated computerized medical equipment, software or other related or similar materials which are intended to be used for diagnosis, prevention, monitoring, treatment of disease [1, 4-8]. Although, the medical devices provide immense benefits to the patients, but the use of medical devices may also lead to some significant potential risks, sometimes life threatening [1, 7]. The risks that are associated with the use of medical devices include harmful effects, in particular, on the patients/users/healthcare professionals, interactions with other substances, certain contraindications and malfunctions. The risks can also include falsifications, technical defects and reduced efficacy $[2,6,9-13]$. This makes it essential to have a regulatory program to monitor these associated adverse effects.

Materiovigilance deals with the identification, collection, reporting estimating the undesirable occurrence and the possible management of adverse events associated with the use of medical devices, thus promoting patient health by preventing its recurrences $[1,5,7]$. The Materiovigilance program of India was launched on 6 July 2015 at the Indian Pharmaceutical Commission, Ghaziabad by DCGI [1, 2, 5] in order to track the medical devices and the associated adverse effects to ensure the safety, provide awareness, generate data, and promoting the patient safety [5, 7].

Scope and objective of materiovigilance

The principle purposes of Materiovigilance are

- To improve the protection of health safety of the patients, users and others by reducing the frequency of an incident $[2,3]$.

- To examine the proposed framework, and its implication, for the Indian medical device vigilance system to global harmonization, i.e., Global Harmonization Task Force (GHTF) [6].

- To bring solutions for the advancement of the use and productivity of the equipment [14].
- To create a nationwide system for patient safety monitoring.

- To analyze the risk benefit ratio of medical devices used [15].

- To generate evidence-based data on the safety of medical devices.

- To support Central Drugs Standard Control Organization (CDSCO) in the decision-making process on the use of medical devices.

- To communicate safety information on the use of medical devices to various stakeholders to minimize the risk.

- To emerge as a national center of excellence for materiovigilance activities.

- To collaborate with other healthcare organizations and international agencies for the National Coordination Centre exchange of information and data management $[4,7]$.

- To sensitize manufacturers, importers, distributors of medical devices, including all healthcare stakeholders for better understanding of medical device standards for promotion of patient safety and strengthening of materiovigilance system.

- To aware stakeholders about the need and significance of medical devices adverse events (MDAE) reporting $[15,16]$.

\section{Methodology}

The publications, events related to medical devices and their adverse effects on Google, Google Scholar, PubMed, and Science Direct were searched with the search keywords such as "medical devices," "adverse effects associated with medical devices," "materiovigilance", "case study related to medical devices" and in combination with each other with no particular time restriction in order to get a holistic and comprehensive view of the research done on this topic so far.

\section{Materiovigilance program of India (MvPI)}

In India, medical devices are classified as drugs and are regulated by drugs and cosmetic act and rules $1945[6,7,17]$. The MvPI is required to regulate the quality, efficacy, safety and availability of medical devices. The medical device rules, 2017 was brought to regulate the manufacture import, sales, distribution of medical devices and came into force from 1 January 2018 [18]. The Central Licensing Approving Authority in 
October 2005, declared 10 devices to be considered as drugs. Medical devices which are classified as drugs include cardiac stents, drugs eluting stents, contraceptive implants, catheters, bone cement, i.e. cannula, intraocular lenses etc. [6, 15].

The MvPI aims at monitoring adverse events associated with the medical devices (medical device associated adverse events). In this program, the IPC functions as a national coordinating center and CDSCO as a regulator (fig. 1). The MvPI includes all private and public health care delivery system as well as the e-reporting system [2]. The MvPI was approved by Ministry of Health and Family Welfare on $10 / 2 / 15$ and it was launched on $06 / 7 / 15$ by DCGI at IPC, Ghaziabad, India $[1,5,19]$.

\section{Classification of medical devices}

Medical devices are classified by each regulatory authority in their own way. In general, the basis for medical devices are classified as on the basis of the risk associated with the medical device, manufacturers' intended purpose for the device and the device's indications for use $[6,16]$.

Classification of medical devices by the United States Food and drug administration (USFDA)

USFDA has classified medical devices into three classes on the basis of level of control necessary to assure the safety and effectiveness of the device and on the basis of information about marketing requirements and has grouped them into 16 medical specialties (table 1) [6].

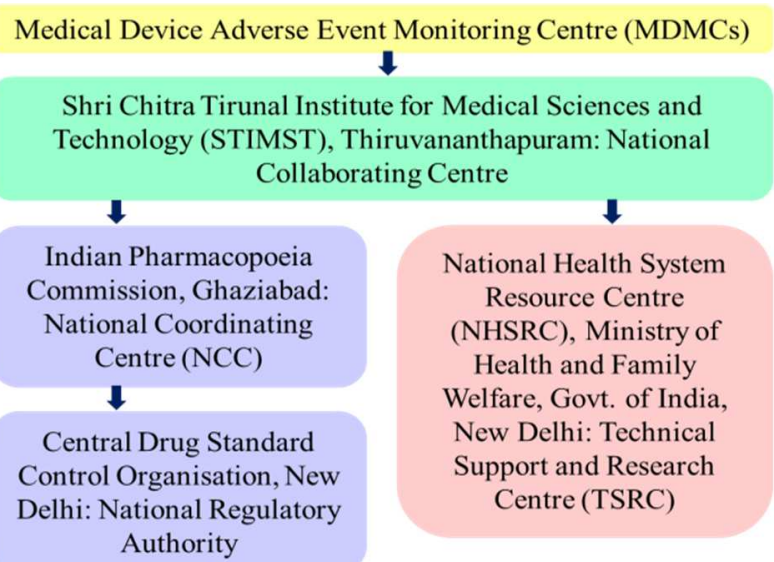

Fig. 1: Organizational structure of materiovigilance program of India [7]

Table 1: Classification of medical devices on the basis of USFDA

\begin{tabular}{lll}
\hline Class & Name of class & Examples \\
\hline Class I & General controls & Elastic bandage and examination gloves \\
Class II & General controls and special controls & Infusion pumps, surgical drapes, and ultrasound imaging systems \\
Class III & General controls and premarket approval & Heart valves, and silicone gel-filled breast implants \\
\hline
\end{tabular}

In addition to other things, the class to which your device is assigned determines the type of premarketing submission required for USFDA clearance to market. If the device is classified as Class I or II and not exempted, a $510 \mathrm{k}$ will be required for marketing. All devices classified as exempt face the limitations on exemptions. A Premarket Approval Application (PMA) will be required for class III devices unless the device is a pre-amendment device (on the market prior to the medical device amendments in 1976, or equivalent to such a device) and PMA's have not been called for. In such a condition, a $510 \mathrm{k}$ will be the route to market $[6,20]$.

Classification of medical devices by the therapeutic goods administration (TGA)

The TGA has classified medical devices into five classes on the basis of a set of four classification rules: invasive, non-invasive, active, and special type of devices, which are discussed in table $2[6,15]$.

Table 2: Classification of medical devices on the basis of TGA

\begin{tabular}{lll}
\hline Class & Risk & Examples \\
\hline Class I & Low risk & Surgical microscopes and examination lights \\
Class IIa & Low to medium risk & Electrical acupuncture and warming blankets \\
Class IIb & Medium to high risk & Infant incubators and external defibrillators \\
Class III & High risk & Heparin-coated catheters and biological heart valves \\
Class IV & Active implantable medical device (AIMD) & Contraceptive intrauterine devices \\
\hline
\end{tabular}

Classification of medical devices by the Medicines and Healthcare products Regulatory Agency (MHRA)

Likewise, the MHRA has classified medical devices into different groups as $[6,21]$ :

- General medical devices
- AIMDs

- In vitro diagnostic medical devices

According to MHRA the general medical devices were then further classified into four classes as described in given table $3[7,21]$.

Table 3: MHRA classification of general medical devices

\begin{tabular}{llll}
\hline Class of device & Risk level & Requirements & Example \\
\hline Class I & Low risk & Premarket notification & Dressings \\
Class IIa & Low-medium risk & Certification by notified body & X-ray film \\
Class IIb & Medium-high risk & Certification by notified body & Blood bags, contact lens care products \\
Class III & High risk & Certification by notified body & Bone cement, cardiac stents \\
\hline
\end{tabular}

In India medical devices are not classified on the basis of risk in comparison to the regulated countries. Rather, the 10-device category of medical devices has been notified to be regulated as drugs [6]. 
Table 4: Risk classification according to the New Zealand regulations

\begin{tabular}{lll}
\hline AIMD & Risk & Implantable pacemaker \\
\hline Class III & High & Drug eluting cardiac stents \\
Class IIb & Medium to High & Ventilators, orthopedic implants \\
Class IIa & Medium to Low & Hypodermic needles, suction equipment \\
Class I sterile & Low & Sterile dressings, non-medicated \\
Class I measuring & Low & Volumetric urine bag \\
Class I basic & Low & Reusable surgical instruments \\
\hline
\end{tabular}

\section{Risk classification according to the New Zealand regulations}

The New Zealand Regulations have five risk classes and two subclasses by following the principles of the GHTF. Based on the basis of the potential risk posed by the medical device when used as intended by the manufacturer, these are classified into different classes as shown in table 4 [22].

\section{Adverse event reporting}

Adverse event reporting system helps in reporting the adverse events associated with the medical device. It is considered as an important tool through which all types of adverse events related to the medical device can be reported $[3,6,16]$. Reporting the adverse event will lead to the protection and improvement of health and safety of the patients or users. It gives us a way to prevent the future repetition of the adverse event to a particular device. All types of adverse events, whether serious, non-serious, adverse events which are pre-known or the incidents which are unknown $[4,5,19,23]$.

The guidance to the manufactures for reporting of adverse events associated with devices has been provided by GHTF. It gives the information on how to handle the information of the adverse event and to decide whether an event is reportable or not on the basis of information available [6, 16]. According to FDA's regulation of medical device reporting, both the manufacturer as well as the importer is required to report the serious adverse events or the malfunctions and also it requires users to report the serious incidents associated with medical devices [6].

\section{What to report}

Any type of adverse incident or event associated with the medical device can be reported. It can be serious, non-serious, pre-known, unknown. It can also be related to the inadequate description of the device and associated risks to the users. Inaccuracy in labelling as well as any malfunction can also be reported. Incidents can be of any type, it can be a frequently occurring event or a rare one, and both the types can be reported. Risks associated with the previous use to the patient can also be provided in the MDAE's reporting form $[1,5$, 13, 23-26].

\section{Where to report}

All the health care professionals, patients, users can report the MDAE's to the Sree Chitra Tirunal Institute of Medical Science and Technology (SCTIMST) or National Coordinating Centre (NCC). The medical device adverse event reporting form can be filled and then it can be sent to SCTIMST, NCC or other stakeholders [5, 27]. The form can also be directly sent through email to mvpi@sctimst.ac.in [28].

\section{How to report}

To report the medical device-associated adverse event, medical device adverse event reporting form can be downloaded from the official website of IPC www. ipc. gov. in $[19,29]$. It is a twopage form which is prepared by MvPI and contains all the information regarding the adverse event, the details of the patient, details of the device for which the adverse event is to be reported, the details of the reporter and the regulator (www.cdsco.nic.in/writereaddata/ MDadverseevent.pdf). The adverse event can also be reported by using the helpline number provided by NCC-PvPI which is 1800-180-3024. The reporter can call on this helpline number on weekdays from 9:00 am to 5:30 pm [5, 23, 29-31].

\section{Reporting criteria for adverse drug reaction found}

The reporting criteria include the following:

- If an event has occurred, and the manufacturer becomes aware of the information.

- If it is assessed that the manufacturer's device is associated with the event based on the opinion from the available information.

The FDA requires the manufacturer to report malfunctions and events occurring due to user errors as well as due to any defect in the medical device. The TGA also requires the sponsor or manufacturer to report malfunctions [6]. CDSCO $[18,19]$ must also report events that do not require to be reported under regulations so that trends or patterns of their occurrence can be monitored.

\section{Not-reportable incidents or events}

The regulated countries, along with India [6], defined not-reportable events similarly with few exceptions. The following events $[6,16,32]$ are exempted from reporting in all countries:

- If the deficiency of a device is found by the user before its use and no serious injury has occurred.

- If the root cause of the adverse event is due to a patient's preexisting condition.

- If the shelf life or service life of the device was exceeded before its use by a patient.

- If the deficiency had a negligible likelihood of causing death or serious injury and had been established and documented as acceptable after risk assessment.

- If the side effects are expected and foreseeable from the manufacturer's labelling, are clinically well known and are documented in the device master record, with an appropriate risk assessment.

- If the adverse event was caused by abnormal use.

\section{Reporting time frame}

Although the report ability of the adverse event is uncertain, the GHTF $[6,16,32]$ requires immediate reporting by the manufacturer of unanticipated death, serious injury and all other reportable events as soon as possible. If the adverse events are reported within the first $2 \mathrm{w}$ based on an incomplete investigation, it may require a next follow-up report. The FDA [6] requires reporting of events not only by the manufacturer, whether domestic or foreign but also by the user facility and distributor. The manufacturer must submit four reports depending on the event reported: first, $30 \mathrm{~d}$ reports for death, serious injury, or malfunctions; second, $5 \mathrm{~d}$ reports for events requiring immediate remedial action (FDA form 3500A); third, baseline report (FDA form 3417) to provide basic data on the device, subject to MDR report ( 30 or $5 \mathrm{~d}$ ); and finally, annual certification (FDA form 3381) [16, 32].

In Australia, although it is the manufacturer who must assess an incident, the sponsor is held accountable for forwarding information about events to the manufacturer and then for forwarding the results of any analysis to the TGA. Hence, the reporting time frame for reporting adverse events is as follows [6]:

- Sponsor or manufacturer: they must report on form MDIR01 the following events: 
- Death or serious deterioration in the state of health within $10 \mathrm{~d}$.

- Near adverse event or event that did not result in death or serious injury within $30 \mathrm{~d}$.

- Serious public health menace or aftercare and an event that requires expeditious remedial action within $48 \mathrm{~h}$.

- Complaints, malfunctions, and adverse events for class III and AIMD devices that have been reported for the year July 1, and October 1 following the entry of device in the Australian Register of Therapeutic Goods (ARTG) and for the subsequent $3 \mathrm{y}$.

- Voluntary reporting by users of a suspected health hazard created by a medical device, on form UDIR01 [6, 33].

Under Incident Report Investigation Scheme of the TGA in United Kingdom, the manufacturer is required to report within the time frame the following reports upon becoming aware that an event has occurred and one of its devices has caused or contributed to the incident [6]:

- Serious public threat within $2 \mathrm{~d}$ after the date of awareness.

- Death or serious deterioration in the state of health within 10 elapsed calendar days after the date of awareness.

- Other incidents, immediately after assessing the link between the device and the event within 30 elapsed calendar days.

- Manufacturer's written acknowledgment of user reports from the MHRA to manufacturer within 3 working days of receiving user report.

- Voluntary reports may be submitted at any time and maybe on the events other than death, serious injury, or malfunction as defined.

The manufacturer's incident report form should be used for beginning, follow-up, and final incident reports. If the report is made orally, it should be followed by a written report as soon as possible and a statement to the effect that the report is made by the
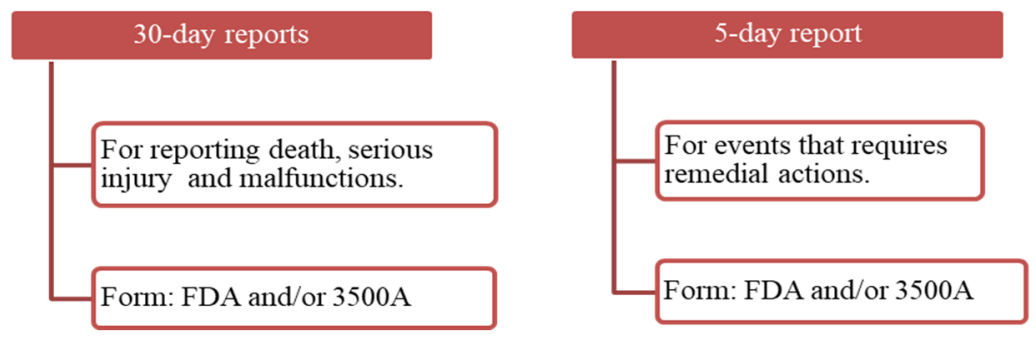

Fig. 2: Mandatory reporting requirements for manufacturers
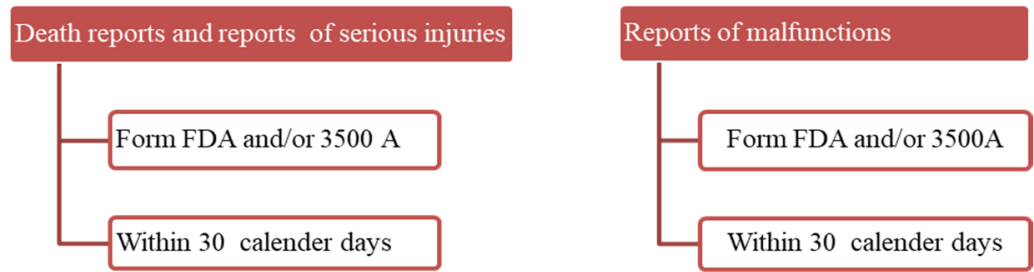

Fig. 3: Mandatory reporting requirements for importers [28]

\section{Vigilance exchange program or vigilance reporting}

National Competent Report System (NCRS) or vigilance exchange program provides guidance/procedures on how to handle the exchange of the two types of information $[6,35,36]$.

- Highly sensitive/Confidential information

- Public/Non-confidential information manufacturer without prejudice and does not imply any admission of liability for the incident or its applicability. The manufacturer or authorized representative must submit an initial incident report to the MHRA for record and evaluation, followed by final reports.

\section{Types of reports}

According to FDA, depending on the reporting time frame, the types of reports that are to be submitted by a manufacturer are of five following types $[6,16,32]$ :

- 30 d report: It involves the submission of reports within $30 \mathrm{~d}$ after the occurrence of the event. Information about death, serious injuries, any malfunction and adverse event reporting through complaint information is reported.

- 5 d report: in some cases, the report needs to be submitted within $5 \mathrm{~d}$ after the day you became aware of the need to submit such a report. This report is required to be submitted for certain serious, unexpected event which requires immediate remedial actions [6, 34].

- Baseline report: when an event for a device model or family is to be reported for the first time [6]. It can be of two types:

- Model type: one report for each model,

- Device family type: one report for all model in that family.

- Supplemental report: it is the follow-up report, which is to be submitted within 1 mo after receipt of additional information.

- Annual certification: this has to be submitted during the 12 mo period. The certifying officials assigned by the manufacturer should sign the certification statement for his identified organizational component/site to minimize the reporting errors $[6,16]$.

In the United Kingdom, the adverse event can be reported by submitting the reports in different forms such as an initial report, periodic summary report, trend report and final report. Whereas CDSCO in India has classified the reports as an initial report and final and/or trend report depending on time and date of report [6]. 
manufacturer's report and the NCRS should be distributed within 14 $\mathrm{d}$ after the event has reported by the manufacturer.

\section{Records}

Records are required to be maintained for each batch of a medical device. It gives information about the product, including its manufacturing, distribution and other relevant data. In order to aid MDR regulations, manufacturers are required to maintain certain written procedures to evaluate the information regarding report ability of an event, protocols that need to be followed during the investigation, for all reports that are submitted to FDA regarding MDR, etc. The records must contain all the history, reported incidents reported about each device as well as the actions taken should also be a part of that record. In case of an event, the records are required to be kept for $2 \mathrm{y}$ or for a period equivalent to the suspected life of that device. The records must provide a detailed evaluation of each event report, user complaints, post-marketed clinical follow-up and so on. These should be maintained throughout the lifetime of the medical device $[6,37]$.

\section{Enforcement actions}

The following enforcement actions have been incorporated by TGA, to ensure the supply of only safe, effective and quality medical devices in the market and for the maintenance of the vigilance program [6, 38]:

\section{- There are penalties for offenses}

- Cancellation of the medical device or, in some cases, suspension of a medical device from the ARTG

- Recall of medical devices.

For the regulation of medical devices, a number of enforcement actions have been described under the Consumer Protection Act 1987, Medical Device Regulations 2002 and General Product Safety Regulations 2005 [6].

The CDSCO should incorporate the enforcement actions in case of a breach of regulations and should also include a recall system.

\section{Medical device regulations 2002}

Medical Device Regulations 2002 has been made on May 20, 2002, and came into force on June 13, 2002. Under section 2 of the European Communities Act 1972, the Secretary of State is a Minister is designated for the purpose of section 2 , to take measures relating to medical devices, with the powers conferred by section 56 and of Finance Act 1973, i.e. in exercise with the consent of the Treasury, in exercise of the power under section 11 and 27 and in exercise of all the power after consultation in accordance with section 11 enabling him in that behalf, of Consumer Protection Act 1987, with the organization that appears to be representative of interest which are markedly affected by these Regulations and other persons which he considers appropriate and with the Health and Safety Commission [3].

\section{Recall of devices}

While marketing a device, the manufacturer, distributor, or consumer might report complaints as some quality defects. If a complaint about a defect is not justified, then it is considered a failure of the quality system and immediate corrective action is undertaken by a product recall.

The guidelines of MHRA $[6,39]$ and GHTF $[6,16]$ have termed recall a Field Safety Corrective Action (FSCA) to reduce the risk of harm to patients, operators, or others or to minimize the reoccurrence of the event. The FSCA would include the following actions:

- Return of a medical device to the manufacturer or its representative (which is termed recall).

- Device modification.

- Device exchange.

- Device destruction.

- Advice is given by the manufacturer regarding the use of the device.
The FSCA could reckon on different gait such as the return of a medical device to the supplier; device modification, exchange or destruction; retrofits by the purchaser of manufacturer's modification or design change [6]; and any advice being given by the manufacturer on the use of the device. However, the manufacturer must distribute a Field Safety Notice (FSN) by appropriate means such as by confirmation of receipt. The FSN itself should include the following items:

- A clear title like "Urgent Safety Notice" on the notice itself, on the envelope, if sent by mail, and as the subject line if sent by email or fax

- The intended audience: a clear statement about the intended recipient of the notice

- A concise description of the subject device (model, batch, or serial number)

- The reasons for the FSCA are explained by the certain factual statement

- A clear description of the hazards associated with the specific failure of the device and, where appropriate, the likelihood of occurrence, being mindful of the intended audience

- The recommended action to be taken by the recipient of the FSN

- Time frames by which the action should be taken by the manufacturer and user, where appropriate

- Designated contact point for the recipient of the FSN to use to obtain further information.

The notice must also include a request to inform customers or patients who received the product. The recalls have been classified on the basis of associated relative health hazard by both FDA and TGA as follows:

- Class I: where severe adverse health consequences or death are likely.

- Class II: where temporary or medically reversible health consequences are likely.

- Class III: where use or exposure to the offending product will not likely cause adverse health consequences.

Class I or class II recalls are considered to be urgent safety-related recalls, whereas class III recalls are considered to be routine nonsafety-related recalls. In addition, the TGA has classified recalls on the basis of the two-phase as premarketing and postmarketing phases $[6,40]$.

\section{Premarketing phase}

- The manufacturer submits a device for assessment along with plans to monitor the performance of devices in use along with the response to any difficulty that may occur to the notified body.

- The notified body conducts a conformity assessment for approval. In case of acceptance, 'CE' mark to the device will be certified to the manufacturer and the notified body also approves the system for monitoring the device's performance and safety.

- Medical Devices with 'CE' marking can be recognized mutually across all the European Union (EU) member states [2, 41].

\section{Post marketing phase}

- All the adverse events are monitored by the manufacturer during the use of their device and necessary action is taken which is also known as FSCA for the reduction in the risk of death or serious fall in the state of health associated with the use of a medical device that is placed in the market. [MEDDEV 2 12-1 rev. 8 Vigilance].

- The adverse incident report related to the device is monitored by the competent authorities in each state (EU member) in their own country, along with the manufacturer's investigation and responses.

- Materiovigilance is designed to generate information for the identification of the problems related to the use of medical devices for the facilitation of the development of safety devices $[2,4,41]$. 


\section{Applications of materiovigilance}

- Diagnosis, monitoring, prevention, treatment, or mitigation of disease or compensation for an injury.

- Improvement in design and efficiency of medical devices.

- Reporting and investigation of the medical device-associated adverse events.

- Implementation of corrective actions to prevent adverse events in future $[1,6,27]$.

- Investigation, replacement, modification, or support of the anatomy or a physiological process.

- Supporting or sustaining life.

- Control of conception.

- Disinfection of medical devices.

- Providing information for medical purposes by means of in vitro examination (such as reagents, calibrators, sample collection kits, control materials and related instruments) of specimens derived from the human body [6].

\section{Medical device tracking}

Medical device tracking has been included by the FDA as one of the post-marketing activities in order to track the device from the time of its manufacture up to the end-user [6]. Medical device tracking helps in locating the device in case of any defect or problems with the device $[6,28,34,42]$. According to the FDA Act, there are certain devices which require tracking such as implantable devices, lifesustaining or life-supporting devices, the failure of such devices will result in serious consequences $[5,6,28]$. The regulations implemented for the tracking of medical devices became effective on 29 august 1993 and can be found in 21 CFR part 8216 [43].

The manufacturer is required to submit the information regarding the medical device whether the device has not or has been distributed to a patient within a period of 3-10 d [6]. The manufacturer needs to establish a written standard operating procedure for tracking the medical device which includes methods and other information regarding the tracking of the medical devices $[28,43]$. The tracking is generally performed for any type of class II and class III devices.

TGA has developed an Implantable Medical Device Tracking Subcommittee (IMDTS) for tracking of patients with implantable medical devices. However, in Europe, the Adverse Incident Tracking System (AITS) has to be followed. The adverse events can be categorized into one of the following investigational categories according to the revised MHRA directives [6]:

- Urgent in-depth (issue medical device alert).

- In-depth

- Standard

- Information

- Other

In India, a lot number or a batch number is assigned to each device in order to make the tracking process easier. While assessing the link between the device and the adverse event, the manufacturer must take into account the following details:

- Opinions from health care professionals

- Previous similar events

- Complaints trends

- Other information held by the manufacturer.

\section{Clinical Investigation or case study about materiovigilance}

Mahajan et al., reported the impact of manufacturer advisories and FDA recalls of implantable cardioverter-defibrillator generators in paediatric and Congenital Heart Disease (CHD) patients from the year 2000-2005, it was found that about $25 \%$ of total patients had recalled devices and a significant proportion of patients underwent explantation. All these complications, though infrequent have important medical as well as psychological impacts and need to be monitored [44]. Beydon et al., studied adverse event reports associated with the medical devices used in anesthesia and intensive care in France. There was about 1004 adverse event reports in the year 1998 and about $11 \%$ cases were classified as serious and $2 \%$ deaths were also reported. There were several causes of failure of medical devices, the leading ones were user errors, quality control problems during the production of the devices and the design fault. 32\% of all reports in France was accounted for anesthesia in 1998. While $98 \%$ of the incidents were not fatal, but they required further examination as the problems with them were same as in the fatal incidents [45]. Laskey et al., performed an analysis of implantable cardiac device reliability from 2003-2007 in the United States. Data reports of devices such as Implantable Cardiac Defibrillators (ICDs) and Cardiac Resynchronization Therapy Defibrillator (CRT-D) implants, explants, and returned devices were analyzed. A statistically significant decrease in implantable cardiac device explantation was observed from year 2003-2007. However, the explantation rates of CRT-D devices remained significantly higher than ICD devices [46]. Golder et al., studied the failure or success of search strategies to identify adverse effects of medical devices and it was found that 51 were included on MEDLINE and 55 were included in EMBASE. Seven of EMBASE were found to be duplicates. Hence, creating a search filter for adverse effects of medical devices is reasonable and should be a research priority [47]. Dieffaga et al., performed a study on the materiovigilance and improvement of the maintenance of the biomedical equipment by the implementation of strategies for the use of equipment. This report was obtained from the case study of the hospital Gabriel Toure of Mali. There were 3 persons able to select the parameter for the use of equipment. 11 persons handle equipment of sterilization and 5 technicians handle radiographies. Only 8 agents move the equipment off after work. 18 agents did not carry out maintenance of material after use. They recommend an internal alteration of the service of maintenance, the programming of regular supervision, the development and the application of the management tools of maintenance [14]. Brockton et al, studied about the adverse events of medical devices from Emergency Departments (ED). There were an estimated 454, $383 \mathrm{ED}$ visits in a 12 mo period in the United States for an adverse event related to the medical device. Around 5350 cases contained two medical devices. The cases included many types of injury diagnoses. The most commonly injured site was the lower trunk $(15 \%)$, followed by the finger (13\%), and the eyeball $(11 \%)$. About $13 \%$ of the total number involved patient hospitalization after ED evaluation. A planned collection of more comprehensive data will allow for appropriate public health interventions [48].

\section{CONCLUSION}

In a few recent years, the use of medical devices is found to be very frequent by medical practitioners all over the world. Despite that, there are no substantial rules and regulations to protect the patients from aversive events related to the use of medical devices. Materiovigilance program is a good initiative by the different countries to ensure the safety of medical devices among the device users globally. This is requisite that emphatic implementation of this program will indemnity the safety of device users or patients. This program will be also significantly reducing the risk related to the use of medical devices by preventing the reduplication of aversive effects.

\section{ACKNOWLEDGMENT}

We acknowledge All India Council of Technical Education (AICTE), Government of India, New Delhi for providing scholarship to the first and second author.

\section{AUTHORS CONTRIBUTIONS}

This review article was initiated and designed by Pratibha Chauhan, Aalia Zarreen, and Mohammad Kashif Iqubal. All the work has been equally contributed by them. The work was overseeing by Mohammad Kashif Iqubal. 


\section{CONFLICTS OF INTERESTS}

The authors declare no conflict of interest there are no conflicts, financial or otherwise.

\section{REFERENCES}

1. Rani S, Singh K. Materiovigilance: an emerging discipline. Int J Sci Res 2018;7:15-6.

2. Materiovigilance in EU. Lambda therapeutic research ltd; 2019. https://www.lambda-cro.com/materiovigilance-in-eu/ [Last accessed on 26 Oct 2018]

3. Madhusudhan SK, Shashikala GH. Evaluation of knowledge, awareness, and practice of pharmacovigilance among practicing doctors. Asian J Pharm Clin Res 2016;9 Suppl 3:283-6.

4. Sivadasan S, Sellappan M. A study on the awareness and attitude towards pharmacovigilance and adverse drug reaction reporting among nursing students in a private university, Malaysia. Int J Curr Pharm Res 2015;7:84-9.

5. Kumar P, Kalaiselvan V, Kaur I, Thota P, Singh GN. Materiovigilance programme of India (MVPI): A step towards patient safety for medical devices. Eur J Biomed Pharm Sci 2016;12:497-501.

6. Gupta P, Janodia MD, Jagadish PC, Udupa N. Medical device vigilance systems: India, US, UK, and Australia. Med Devices (Auckl) 2010;3:67.

7. Meher BR. Materiovigilance: an Indian perspective. Perspect Clin Res 2018;9:175-8.

8. Johnson JA. FDA regulation of medical devices. Congressional research service; 2016. p. 1-45.

9. https://www.globalbsg.com/iwe-event/2nd-annualpharmacovigilance-drug-safety-summit/ [Last accessed on 22 Nov 2018].

10. McCulloch $\mathrm{P}$. The EU's system for regulating medical devices. $\mathrm{Br}$ Med J 2012;345:1-2.

11. Medicines and Healthcare products regulatory agency. Annual Report Accounts 2010/11;1-71. Available from: https://assets.publishing.service.gov.uk/government/uploads/ system/uploads/attachment_data/file/247348/1195.pdf [Last accessed 22 Nov 2018].

12. Postmarket surveillance under section 522 of the federal food, drug, and cosmetic act. Guidance for Industry and Food and Drug Administration Staff. USFDA; 2016. p. 1-22. Available from: https://www.fda.gov/downloads/MedicalDevices/DeviceRegula tionandGuidance/GuidanceDocuments/ucm268141.pdf [Last accessed 12 Dec 2018]

13. Jankar BB, Devesh D, Gosavi. Adverse drug reaction of lithium carbonate-a review. J Crit Rev 2017;4:1-6.

14. Dieffaga T, Sanogo M, Maiga S. P360: materiovigilance and improvement of the maintenance of the biomedical equipment by the implementation of strategies for the use of equipment: case study of the hospital Gabriel Touré of Mali. Antimicrob Resist Infect Control 2013;2 Suppl 1:P360.

15. Wathoni N, Alfauziah TQ, Rantika N. Evolution of contraceptive implants: a review. Int J Appl Pharm 2018;10:16-22.

16. Principles of medical devices classification. The global harmonization task force; 2006. Available from: http://www.imdrf.org/docs/ghtf/final/sg1/technical-docs/ ghtf-sg1-n15-2006-guidance-classification-060627.pdf [Last accessed on 01 Nov 2018].

17. A guidance document for medical devices. Central drugs standard control organization; 2018. Available from: http://www.cdsco.nic.in/writereaddata/Guidance\%20Docume nt\%20ipv.pdf [Last accessed on 26 Nov 2018]

18. Classification of medical devices and in vitro diagnostic medical devices under the provisions of the medical devices rules. Drugs Controller General, India; 2017. Available from: http://www.cdsco.nic.in/writereaddata/Classification\%20wis e\%20list\%20of\%20MD\%20and\%20IVDs17.pdf [Last accessed on 01 Nov 2018].

19. Masurkar P. A need of better pharmacovigilance system in India. Asian J Pharm Clin Res 2017;10:22-4.

20. Classify Your Medical Device. USFDA; 2018. Available from: https://www.fda.gov/medicaldevices/deviceregulationandgui dance/overview/classifyyourdevice/ucm2005371.htm [Last accessed on 22 Jan 2019]

21. Medicines and Healthcare products regulatory agency. Available from: https://www.gov.uk/topic/medicines-medical-devicesblood/medical-devices-regulation-safety [Last accessed on 13 Feb 2019].

22. Risk classification of medical devices. New Zealand Medicines and Medical Devices Safety Authority; 2011. Available from: https://medsafe.govt.nz/regulatory/devicesnew/37RiskClassification.asp [Last accessed on 01 Nov 2018]

23. Kalaiselvan V, Kumar P, Mishra P, Singh GN. System of adverse drug reactions reporting: what, where, how, and whom to report? Indian J Crit Care Med 2015;19:564-6.

24. Kirschgens LA, Ugarte IZ, Uriarte EG, Rosas AM, Vilches VM. Robot hazards: from safety to security. arXiv preprint arXiv: 1806.06681; 2018. p. 1-10.

25. TGA actions after review into urogynaecological surgical mesh implants. Therapeutic goods administration (TGA). Australian Government, Department of health; 2019. Available from: https://www.tga.gov.au/alert/tga-actions-after-reviewurogynaecological-surgical-mesh-implants [Last accessed 10 Jan 2019].

26. Thacker T. Govt plans tweaks in law to make firms liable for defective devices, drugs; 2017. Available from: https://www.livemint.com/Politics/sTQOfekT0SVdqlqo0wu3x L/Govt-plans-tweaks-in-law-to-make-firms-liable-fordefective.html [Last accessed on $21 \mathrm{Feb} 2019$ ]

27. Nagaratnam C, Deepika B, Deepalatha C. Drug utilization pattern study in young adult patients of cutaneous adverse drug reactions. Int J Curr Pharm Res 2018;10:13-5.

28. MDR Adverse Event Codes. USFDA; 2018. Available from: https://www.fda.gov/medicaldevices/deviceregulationandgui dance/postmarketrequirements/reportingadverseevents/mdr adverseeventcodes/default.htm [Last accessed on 19 Dec 2018].

29. Materiovigilance Programme of India (MvPI)-Indian Pharmacopoeia Commission. Available from: http://www.ipc.gov.in/mandates/pvpi/materiovigilanceprogramme-of-india-mvpi.html [Last accessed on 28 Dec 2018]

30. Kaur I, Kalaiselvan V, Kumar R, Kumar P, Singh GN. Reporting of adverse drug reaction under pharmacovigilance programme of India; 2015.

31. The importance of pharmacovigilance-safety monitoring of medicinal products. World Health Organization; 2002. Available from: http://apps.who.int/medicinedocs/ en/d/ Js4893e/ [Last accessed on 28 Dec 2018]

32. Comparison of the device adverse reporting systems in USA, Europe, Canada, Australia and Japan. Global harmonization task force; 2002. Available from: http://www.imdrf.org/docs/ ghtf/final/sg2/technical-docs/ghtf-sg2-n6r3-2002comparison-device-adverse-reporting-systems-020521.pdf [Last accessed on 22 Dec 2018]

33. The Medical Devices Regulations. UK Statutory Instruments; 2002. p. 618. Available from: http://www.legislation. gov.uk/uksi/2002/618/introduction/made [Last accessed on 23 Mar 2019]

34. Medical device manufacturers. Guide to inspections of medical device manufacturers, USFDA; 1997. Available from: https://www.fda.gov/ICECI/Inspections/InspectionGuides/uc m074899.htm [Last accessed on 26 Dec 2018]

35. Guidance on how to handle information concerning vigilance reporting related to medical devices. Global harmonization task force 1999. Available from: http://www.imdrforg/ docs/ghtf/final/sg2/technical-docs/ghtf-sg2-n008r4reporting-guidance-990629.pdf [Last accessed on 27 Dec 2018]

36. Application requirements for participation in the GHTF national competent authority report exchange program. Global harmonization task force; 2009. Available from: http://www.imdrf.org/docs/ghtf/final/sg2/technical-docs/ ghtf-sg2-n38r19-national-competent-authority-reportprogram-090701.pdf [Last accessed on 16 Jan 2018].

37. Medical device tracking guidance for industry and food and drug administration staff. USFDA; 2014. Available from: https://www.fda.gov/downloads/MedicalDevices/DeviceRegu 
lationandGuidance/GuidanceDocuments/ucm071775.pdf [Last accessed on 28 Dec 2018].

38. Recalls, suspensions, cancellations, and tampering of medical devices. Australian regulatory guidelines for medical devices (ARGMD) part 3-post-market, therapeutic goods administration; 2011. p. 317-37. Available from: https:// www.tga.gov.au/sites/default/files/devicesargmd-p3.docx [Last accessed on 29 Oct 2018].

39. Recalls, corrections and Removals (Devices). USFDA; 2018. https://www.fda.gov/medicaldevices/deviceregulationandgui dance/postmarketrequirements/recallscorrectionsandremoval s/default.htm [Last accessed on 18 Nov 2018].

40. Uniform recall procedure for therapeutic goods (URPTG) version 2.1. Therapeutic goods administration; 2019. p. 1-79. Available from: https://www.tga.gov.au/publication/uniformrecall-procedure-therapeutic-goods-urptg-2004-edition [Last accessed on 18 Jul 2019].

41. Parvizi N, Woods K. Regulation of medicines and medical devices: contrasts and similarities. Clin Med (Lond) 2014;14:6-12.

42. Medical Device Overview. USFDA; 2018. Available from: https://www.fda.gov/ForIndustry/ImportProgram/ImportBas ics/RegulatedProducts/ucm510630.htm [Last accessed on 22 Nov 2018].

43. Golder S, Wright K, Rodgers M. Failure or success of search strategies to identify adverse effects of medical devices: a feasibility study using a systematic review. Syst Rev 2014;3:1-6.

44. Beydon L, Conreux F, Le Gall R, Safran D, Cazalaa JB. Analysis of the french health ministry's national register of incidents involving medical devices in anesthesia and intensive care. $\mathrm{Br} \mathrm{J}$ Anaesth 2001;86:382-7.

45. Laskey W, Awad K, Lum J, Skodacek K, Zimmerman B, Selzman $\mathrm{K}$, et al. An analysis of implantable cardiac device reliability. The case for improved postmarketing risk assessment and surveillance. Am J Ther 2012;19:248-54.

46. Inspection of Medical Device Manufacturers: attachment D: summary of tracking requirements. USFDA; 2018. Available from: https://www.fda.gov/MedicalDevices/DeviceRegulation and Guidance/GuidanceDocuments/ucm149673.htm [Last accessed on 28 Nov 2018].

47. Hefflin BJ, Gross TP, Schroeder TJ. Estimates of a medical device-associated adverse events from emergency departments. Am J Prev Med 2004;27:246-53.

48. Mahajan T, Dubin AM, Atkins DL, Bradley DJ, Shannon KM, Erickson CC, et al. Impact of manufacturer advisories and FDA recalls of implantable cardioverter-defibrillator generators in pediatric and congenital heart disease patients. J Cardiovasc Electrophysiol 2008;19:1270-4. 